\title{
Colloidal Aggregation in Microgravity by Critical Casimir Forces
}

Physical Review Letters 109, 248302 (2012)

Authors: S.J. Veen, O. Antoniuk, B. Weber, M.A.C. Potenza, S. Mazzoni, P. Schall, and G.H. Wegdam

\section{Recommended and a commentary by Marjolein Dijkstra, Utrecht University}

In this paper, Sandra J. Veen and coauthors studied the aggregation of colloidal particles by critical Casimir forces using scattering experiments. They performed experiments on charge stabilized fluorinated latex particles in a binary solvent mixture consisting of 3-methyl pyridine and water. Striking differences in the structure of the resulting fractal aggregates as well as in the kinetics was found between microgravity experiments on board of the International Space Station and ground experiments. In microgravity, fractal structures were formed by diffusion limited aggregation with a fractal dimension that decreases continuously with increasing attraction. In experiments conducted on the ground show reaction limited aggregation resulting in one type of aggregate with a constant fractal dimension.

In order to control the interactions between colloidal particles, the authors employed the critical Casimir effect [1]. Critical Casimir forces arise between particles that are suspended in a binary liquid mixture, which is near to its critical point. The confinement of critical composition fluctuations between the surfaces of the colloidal particles leads to Casimir-like long-range forces, which are analogous to the quantumelectrodynamical Casimir forces that result from confined quantum-electrodynamical vacuum fluctuations between two metallic plates [2]. The range and amplitude of the critical Casimir forces are determined by the bulk correlation length of the mixture which depends strongly on the temperature and which diverges upon approaching the critical point. Hence, the critical Casimir forces are extremely temperature-sensitive close to the critical point, and offer excellent reversible control over the colloid interactions. Critical Casimir forces have been measured experimentally by direct force measurements of a single colloid above a flat surface and can be either attractive or repulsive depending on the adsorption preferences of the colloidal particle and the wall [3].

In this paper, the first experimental study of the relation between the strength of attraction and the structure of the aggregates is reported by using the temperaturesensitive Critical Casimir force. Experiments were conducted on samples of charged colloids with a colloid volume fraction of $\sim 10^{-4}$ in a binary solvent mixture of 3methyl pyridine (3MP) and water with a $3 \mathrm{MP}$ weight fraction of $\mathrm{X}_{3 \mathrm{MP}}=0.39$, and four different salt concentrations $0.31,0.79,1.5$, and $2.7 \mathrm{mmol} / 1$ sodium chloride. This solvent mixture has a lower critical temperature of $48^{\circ} \mathrm{C}$. For each sample, the aggregation temperature was determined, and subsequently, the aggregation was studied with increasing temperature, to study systematically the aggregation at increasing particle attractions. Unfortunately, the authors do not report the critical composition of the mixture and the exact temperature differences with respect to the critical temperature. Subsequently, it is shown from light scattering experiments that 
the fractal dimension of the formed aggregates in microgravity decreases from 2.4 (compact clusters) at low temperature to 1.8 (more open structures) at high temperatures. More surprisingly, perhaps, no dependence was observed on the salt concentration in contrast with recent experiments where it was shown that the addition of salt enhances strongly the critical Casimir forces between a single particle and a wall as the electrostatic screening leads to smaller particle-wall distances [4]. On the ground, it was observed that the fractal dimension of the aggregates is $\sim 1.7$, and is independent of the temperature and salt concentration. Interestingly, the scattering data also reveals that the growth of the aggregates in microgravity shows characteristics of the spinodal decomposition process, and that the underlying dynamics resembles diffusion limited aggregation. The aggregation on the ground is strongly affected by sedimentation resulting in reaction limited aggregation.

In this letter, the first experimental study of the relation between attraction strength and the structure of the aggregates is reported by using the critical Casimir effect. The present investigation may prove instrumental in guiding future studies on colloid aggregation, e.g. at different temperature paths and off-critical compositions, where one expects to observe an interplay between critical adsorption, complete wetting, and capillary condensation $[5,6]$.

[1] M.E. Fisher and P.G. de Gennes, C.R. Acad. Sci. Paris B 287, 207 (1978).

[2] H. B. G. Casimir, Proc. K. Ned. Akad. Wet. 51, 793 (1948).

[3] C. Hertlein, L. Helden, A. Gambassi, S. Dietrich, and C. Bechinger, Nature 451, 172, (2008).

[4] U. Nellen, J. Dietrich, L. Helden, S. Chodankar, K. Mygard, J.F. van der Veen, and C. Bechinger, Soft Matter 7, 5360 (2011).

[5] A. Drzewinski, A. Maciolek, A. Barasinski, and S. Dietrich, Phys. Rev. E 79, 041145 (2009).

[6] R. Okamoto and A. Onuki, J. Chem. Phys. 136, 114704 (2012). 